\title{
Regression of an asymptomatic choroidal metasta- sis from breast carcinoma following letrozole ther- apy*
}

\author{
Jayshan Yohendran** and Devinder Chauhan*** \\ **Royal Victorian Eye and Ear Hospital, 32 Gisbourne Street, East Melbourne, Victoria, Australia \\ 3002 \\ ***Vision Retinal Institute, 852 Whitehorse Road, Box Hill, Victoria, Australia 3128
}

<jay@gmp.usyd.edu.au>

Received 28 August 2009; revised version accepted 27 October 2009

\begin{abstract}
Background: Uveal metastases are commonly of breast origin, typically affecting the posterior choroid. Treatment of such uveal tumours can lead to significant ocular side effects.

Methods: We present the clinical and optical coherence tomography findings of a patient with an asymptomatic choroidal metastases.

Results: A 62-year-old lady presented with an
\end{abstract}

asymptomatic uniocular choroidal metastases from breast origin. She was being treated with letrozole, a nonsteroidal aromatose inhibitor, for known pleural metastases. The choroidal lesion was inactive at presentation to our clinic and required no further treatment.

Conclusion: The use of letrozole can lead to choroidal metastasis regression.

Keywords: Choroidal metastasis, letrozole, breast carcinoma, aromatase inhibitors

\section{Introduction}

Uveal metastases are commonly of breast origin, typically affecting the posterior choroid. We report a case of asymptomatic uniocular choroidal metastases from breast origin in a lady who was being treated with letrozole, a nonsteroidal aromatose inhibitor, for known pleural metastases. The choroidal lesion was inactive at presentation to our clinic.

\section{Case Report}

A 62 year old lady was referred to our clinic by her optometrist for an opinion regarding an incidental retinal finding in her right eye. She was seen by the same

\footnotetext{
**MBBS(Hons) MPH BMedSci

***MBBS FRCOphth MD FRANZCO
}

*The patient in this report was seen at Vision Retinal Institute.

There were no grants or funds received by the authors in support of this paper. Nor do the authors have any proprietary interest. The authors have no conflicts or potential conflicts to disclose. 


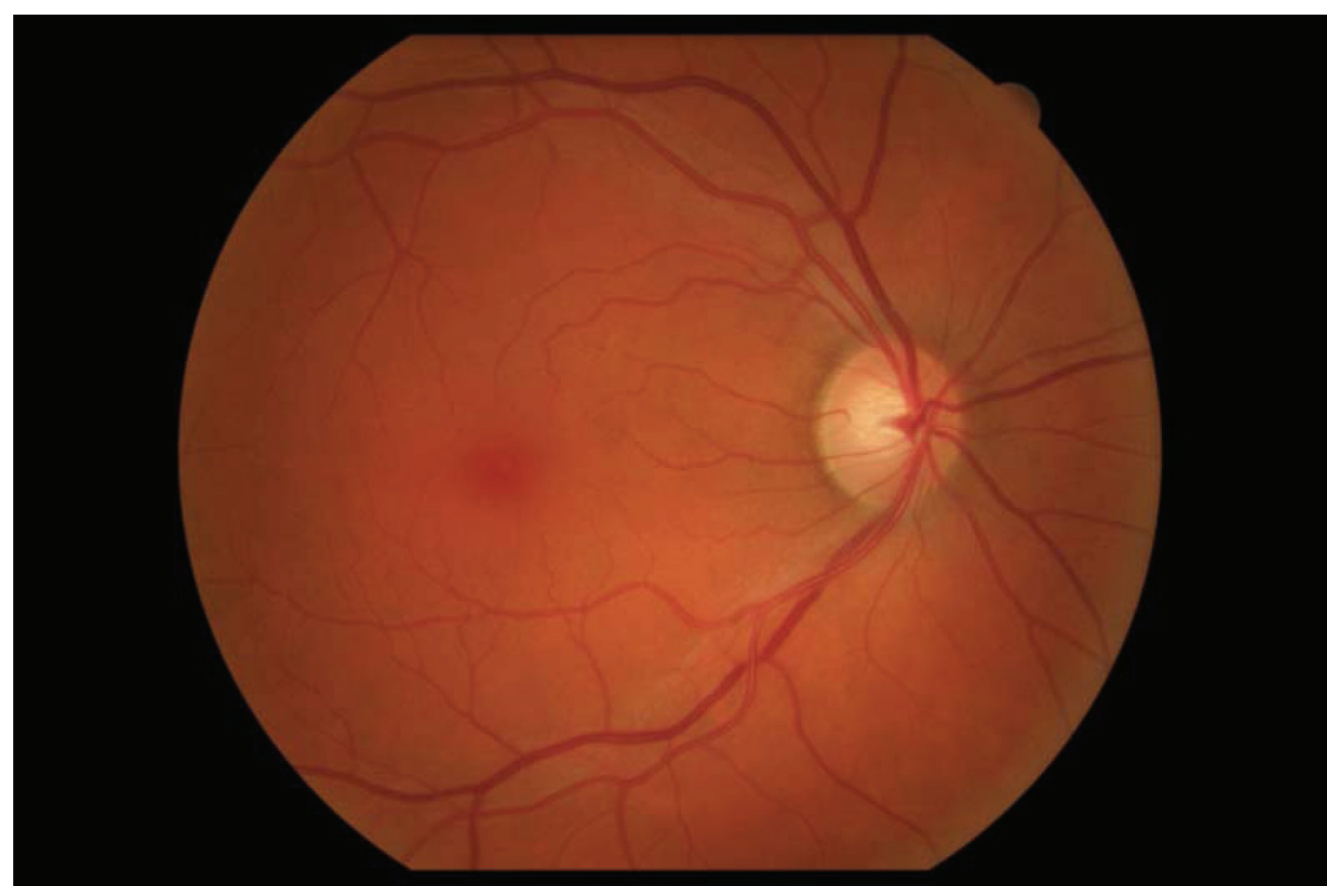

Figure 1: Colour fundus photograph of right eye in February 2005. No indications of retinal disease were present.

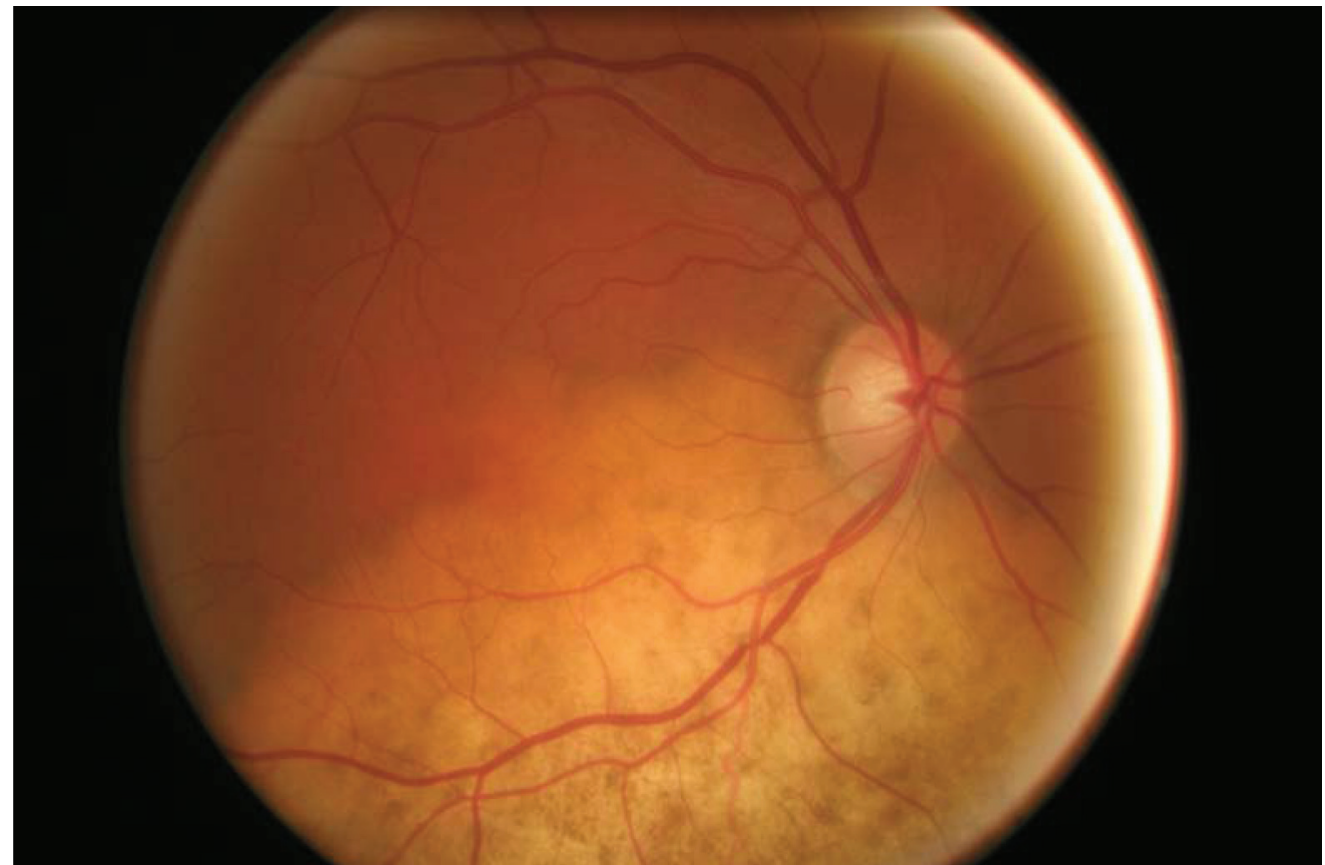

Figure 2: Colour fundus photograph of right eye in May 2007 showing an extensive area of retinal pallor in the inferior macular with areas of altered pigmentation of the RPE.

optometrist approximately two years prior for a routine check. At that visit her right fundus examination was unremarkable. This was documented photographically (Figure 1). At her most recent routine visit, her optometrist was concerned to note a large diffuse pale area in her right inferior macula, extending out to the equator of the eye. Again, this was documented photographically (Figure 2). The patient noticed no visual symptoms in the intervening time between these photographs. A formal Medmont visual field test revealed a corresponding mild supero-temporal defect and a marginally enlarged blind spot (Figure 3). 
The patient was diagnosed with a breast carcinoma in 1995 (T2N1 on TNM staging - tumour sized between 2-5 cm with metastases to ipsilateral regional lymph nodes). She was treated surgically and then with tamoxifen for five years, as per the standard protocol. Approximately 18 months prior to her review in our clinic she developed chest symptoms. She was found to have a pleural effusion as a consequence of metastatic breast carcinoma. Her treatment included letrozole, a nonsteroidal aromatose inhibitor used for hormone receptor positive breast cancer in postmen- opausal women. This treatment is ongoing and has been successful in controlling the pleural disease.

At our clinic her best corrected visual acuity was 6/6 RE and 6/4.8 LE. Anterior segment examination was unremarkable. There were no cells in her anterior vitreous bilaterally and there was no posterior vitreous detachment. Fundus examination of the right eye revealed an extensive area of retinal pallor in the inferior macular with areas of altered pigmentation of the RPE. There were no associated areas of subretinal fluid and no elevation of the choroid or retina on slit-

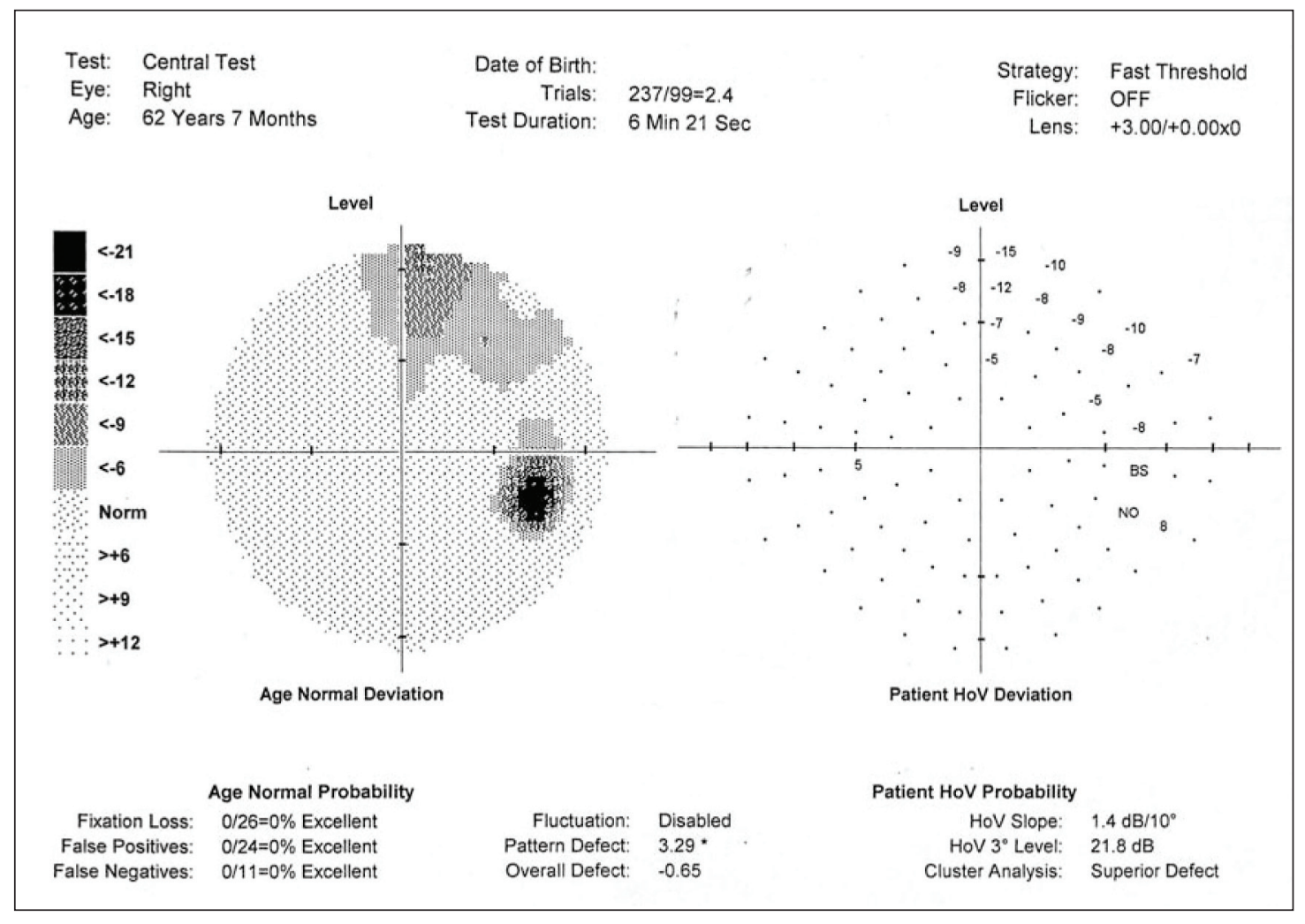

Figure 3: Medmont visual field test showing a mild supero-temporal defect. The blind spot was marginally enlarged on automated analysis.

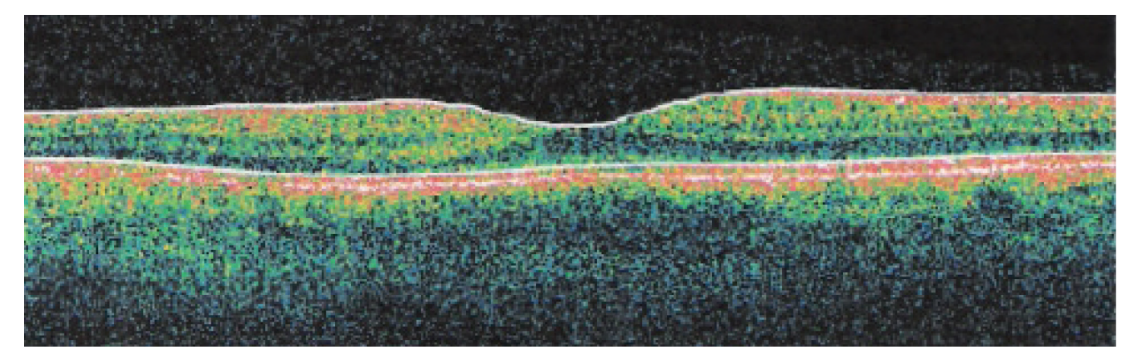

Figure 4: OCT image of right eye showing no areas of subretinal fluid, no elevation of the choroid and no retinal thickening. The scan was taken in vertical orientation and passing through the fovea (the inferior retina is to the left on the figure). 
lamp biomicroscopy. This was confirmed by Zeiss Stratus OCT analysis (see Figure 4). ${ }^{3}$

A diagnosis of presumed regressed choroidal breast carcinoma metastasis was made. As this lesion was inactive, no new treatment was proposed. As the patient was being treated with letrozole for her pleural disease, the plan in regards to the eye was simply for observation. Three months later, she had a scalp lesion removed which was histologically proven to be another metastatic lesion.

\section{Discussion and Conclusion}

Breast cancer is the most common invasive cancer diagnosed in females. The incidence of ocular metastases from breast cancer has been reported between $3-30 \% .{ }^{1}$ The wide range is due to the asymptomatic nature of uveal metastases and the lack of routine ocular screening. Treatment options for such lesions include observation, systemic treatment (chemotherapy, hormone therapy, immunotherapy), radiotherapy (external beam radiation therapy, plaque), local resection, photocoagulation, transpupillary thermotherapy, cryotherapy and enucleation.

Oestrogen plays an important role in the development and growth of breast tumours. Hormone therapy is commonly used to treat oestrogen receptor positive tumours. Traditionally, such patients are treated with tamoxifen, a competitive oestrogen receptor antagonist. Manquez et $a l^{1,2}$ have found that treatment with new third generation aromatase inhibitors, including letrozole, causes regression of choroidal metastases. In their series, 10 of 17 patients with oestrogen receptor positive breast cancer responded dramatically to aromatase inhibitors. Many of the cases regressed after failing to respond to tamoxifen. Aromatase inhibitors act by preventing the peripheral conversion of adrenal androgen to oestrogen, thereby reducing the levels of circulating oestrogen. Such patients avoid the side effects of local treatment like external beam radiotherapy.

Our patient has been reviewed over twelve months after her initial visit at our clinic, and her lesion remains inactive. There has been no change in size of this pale lesion. She has not developed visual symptoms.

\section{References}

1. Manquez ME, Brown MM, Shields CL. Shields JA. Management of choroidal metastases from breast carcinomas using aromatase inhibitors. Current Opinion in Ophthalmology 2006 17(3) 251-6.

2. Manquez ME, Shields CL, Karatza EC. Shields JA. Regression of choroidal metastases from breast carcinoma using aromatase inhibitors. British Journal of Ophthalmology 2005 89(6) 776-7.

3. Arevalo JF, Fernandez CF, Garcia RA. Optical coherence tomography characteristics of choroidal metastasis. Ophthalmology 2005112 1612-1619. 